\title{
Fat deposition decreases diffusion parameters at MRI: a study in phantoms and patients with liver steatosis
}

\author{
Helena S. Leitão • Sabrina Doblas • Gaspard d'Assignies • \\ Philippe Garteiser • Jean-Luc Daire • Valérie Paradis • \\ Carlos F. G. C. Geraldes • Valérie Vilgrain • \\ Bernard E. Van Beers
}

Received: 25 April 2012 /Revised: 19 June 2012 / Accepted: 29 June 2012 / Published online: 1 September 2012

(C) European Society of Radiology 2012

\begin{abstract}
Purpose Assess the effect of fat deposition on the MRI diffusion coefficients in lipid emulsion-based phantoms and patients with proven isolated liver steatosis.

Materials and methods Diffusion-weighted MRI with $11 \mathrm{~b}$ values from $0-500 \mathrm{~s} / \mathrm{mm}^{2}$ was performed in phantoms (fat fractions $0-18 \%$ ) with and without fat suppression
\end{abstract}

\section{H. S. Leitão}

Center for Neuroscience and Cell Biology, PhD Program in

Experimental Biology and Biomedicine, University of Coimbra,

Coimbra, Portugal

H. S. Leitão $\cdot$ S. Doblas · G. d'Assignies · P. Garteiser •

J.-L. Daire $\cdot$ V. Vilgrain $\cdot$ B. E. Van Beers

Laboratory of Physiological and Molecular Imaging of the

Abdomen (IPMA) and Department of Radiology,

University Paris Diderot, Sorbonne Paris Cité, INSERM UMR 773,

University Hospitals Paris Nord Val de Seine, Beaujon,

Clichy, France

\section{Paradis}

Department of Pathology, University Paris Diderot,

Sorbonne Paris Cité, INSERM UMR 773,

University Hospitals Paris Nord Val de Seine, Beaujon,

Clichy, France

\section{F. G. C. Geraldes}

Department of Life Sciences, Faculty of Science and Technology, Center for Neuroscience and Cell Biology and Coimbra Chemistry Center, University of Coimbra,

Coimbra, Portugal

H. S. Leitão $(\bowtie)$

Department of Radiology and IPMA, INSERM UMR 773,

University Hospitals Paris Nord Val de Seine, Beaujon,

100 boulevard du Général Leclerc,

92110 Clichy, France

e-mail: helenasleitao@netcabo.pt and in 19 patients with normal liver $(n=14)$ or isolated liver steatosis $(n=5)$ proven by histopathology. The apparent, pure and perfusion-related diffusion coefficients and the perfusion fraction were measured. Spearman correlation coefficient and Mann-Whitney $U$ test were used for comparisons.

Results A strong correlation between the apparent and pure diffusion coefficients and fat fractions was seen in phantoms. The pure diffusion coefficient decreased significantly in patients with liver steatosis $\left(0.96 \pm 0.16 \times 10^{-3} \mathrm{~mm}^{2} / \mathrm{s}\right.$ versus $1.18 \pm 0.09 \times 10^{-3} \mathrm{~mm}^{2} / \mathrm{s}$ in normal liver, $P=0.005$ ), whereas the decrease in apparent diffusion coefficient did not reach statistical significance $\left(1.26 \pm 0.25 \times 10^{-3} \mathrm{~mm}^{2} / \mathrm{s}\right.$ versus $1.41 \pm$ $0.14 \times 10^{-3} \mathrm{~mm}^{2} / \mathrm{s}$ in normal liver, $P=0.298$ ).

Conclusions Fat deposition decreases the apparent and pure diffusion coefficients in lipid emulsion-based phantoms and patients with isolated liver steatosis proven by histopathology.

Key Points

- Magnetic resonance imaging is increasingly used to quantify hepatic fibrosis.

- Lipid phantoms show inverse correlations between diffusion coefficients and fat fractions.

- The pure diffusion coefficient decreases in patients with isolated liver steatosis.

- Steatosis may be a confounding factor when measuring the liver diffusion parameters.

Keywords Liver · Steatosis · Magnetic resonance imaging · Diffusion-weighted MRI · Phantom imaging

Chronic liver diseases are frequent causes of morbidity and mortality in the Western countries. In a recent screening 
study performed in a general population older than 45 years, liver fibrosis related to unsuspected chronic liver disease was detected in $7.5 \%$ of the subjects and cirrhosis in about $1 \%$ [1]. The most frequent causes of chronic liver diseases encountered were nonalcoholic fatty liver disease (NAFLD), alcoholic liver disease and viral hepatitis C and B [1]. Among these, nonalcoholic fatty liver disease is a major healthcare problem since excessive liver fat is detected in one third of the United States adult population $[2,3]$.

Recently, various imaging methods, including diffusionweighted magnetic resonance imaging, have emerged as potential biomarkers for chronic liver disease [2, 4]. Prior studies have shown that chronic liver diseases are associated with a decrease of the diffusion coefficients. This has been related to progressive fibrosis, inflammation and decreased perfusion [5-9]. Liver steatosis is also frequently observed in patients with liver fibrosis, especially in patients with nonalcoholic and alcoholic liver diseases and viral hepatitis $\mathrm{C}$ and $\mathrm{B}[10,11]$.

However, only few and conflicting data are available regarding the influence of liver steatosis on the diffusion parameters. In most reported studies liver steatosis is considered not to restrict diffusion $[5,12,13]$. Two recent studies performed in patients and rats suggested a relationship between steatosis and diffusion [14, 15]. However, because no liver biopsies were obtained in the patient study, the effect of confounding factors such as fibrosis and inflammation on the diffusion measurements could not be assessed. In the animal study, biopsies were obtained, but a multivariate analysis of the influence of steatosis, inflammation and fibrosis on the diffusion measurements was not performed. Therefore, the purpose of our study was to assess the effect of fat deposition on the MRI diffusion coefficients, in lipid emulsion-based phantoms and in patients with normal liver and with isolated liver steatosis without any other confounding factor as confirmed by histopathology.

\section{Materials and methods}

\section{Lipid emulsion-based phantom study}

The phantoms consisted of 50-ml tubes containing a constant amount of $5 \mathrm{ml}$ of gelatin used as a solidification agent. To obtain the various fat fractions in the phantoms, we progressively increased the added amount of a lipid emulsion of refined olive oil $(16 \%)$ and soybean oil (4\%) (ClinOleic $20 \%$, Baxter, Maurepas, France) in each tube and decreased the added amount of water in parallel. The final fractions of fat in the phantoms ranged from 0 to $18 \%$ (0\%, $3 \%, 5 \%, 7 \%, 9 \%, 12 \%, 14 \%, 16 \%, 18 \%)$. Fat droplets inside the phantoms had a mean diameter of
$0.6 \mu \mathrm{m}$, which is similar to that of fat droplets within the hepatocyte cytoplasm (about $1 \mu \mathrm{m}$ ) [16, 17].

The phantoms were imaged using 1.5 T MR (Intera, Philips Medical Systems, Best, The Netherlands) with a four-element surface coil. Diffusion-weighted magnetic resonance imaging was performed without and with a fat suppression scheme (Spectral Presaturation with Inversion Recovery) using a single-shot echo-planar acquisition and the following parameters: repetition time/echo time 305/ $57 \mathrm{~ms}$, matrix $80 \times 80$ pixels, $250-\mathrm{mm}$ field of view, 3 transverse slices, slice thickness $4 \mathrm{~mm}, 11 \mathrm{~b}$ values $(0$, $10,20,30,40,50,75,100,150,300$ and $500 \mathrm{~s} / \mathrm{mm}^{2}$ ), 20 averages and 3 directions. The acquisition time was $3 \mathrm{~min} 30 \mathrm{~s}$.

\section{Patient study}

This retrospective clinical study was approved by the review board at our institution and informed consent was waived. Patients who underwent magnetic resonance imaging for the assessment of a liver tumour between June 2010 and March 2011 were identified for this study. Inclusion criteria were: age 18 years and older; liver MRI with a multi-b diffusionweighted sequence; histopathological assessment of the tumour and non-tumorous liver parenchyma performed by liver biopsy or after surgical resection. Ninety-seven patients were identified based on the inclusion criteria.

The diffusion-weighted images in the patients were obtained using the same 1.5-T MRI system and surface coil described above for the phantom study. The multi-b singleshot echo-planar diffusion-weighted acquisition had the following parameters: repetition time/echo time $305 / 57 \mathrm{~ms}$, matrix $80 \times 80$ pixels, $320-\mathrm{mm}$ field of view, 3 transverse slices, slice thickness $4 \mathrm{~mm}, 11 \mathrm{~b}$ values $(0,10,20,30,40$, $50,75,100,150,300$ and $\left.500 \mathrm{~s} / \mathrm{mm}^{2}\right), 20$ averages and 3 directions. Image acquisition was obtained with fat suppression (Spectral Presaturation with Inversion Recovery) and free breathing. The acquisition time was $3 \min 30 \mathrm{~s}$.

Exclusion criteria were as follows: motion artefact on the diffusion-weighted images precluding the analysis of the diffusion parameters (7 patients); liver inflammation, fibrosis, cirrhosis or iron overload at histopathological evaluation (71 patients). The final study population consisted of 19 patients, 12 women and 7 men, with a mean age of 50.7 years (range: $24-72$ years for women; $37-74$ years for men). The median interval between magnetic resonance imaging and biopsy $(n=16)$ or surgical resection $(n=3)$ was 14.5 days (range: $0-90$ days).

For the patients included in the study, the non-tumourous liver biopsies were retrospectively reviewed by an experienced hepatobiliary pathologist who was unaware of imaging results. This evaluation confirmed the absence of steatosis, inflammation, fibrosis and iron $(n=14$, normal 
liver) or the presence of isolated liver steatosis $(n=5)$, and grading was performed according to the Brunt classification [18]: three patients had grade 1 steatosis (number of hepatocytes containing fat: 5-33\%), one grade $2(33-66 \%)$ and one grade 3 (> $66 \%$ ).

Image interpretation

Regions of interest were placed on the diffusion-weighted images by one of the authors with 5-year experience in MRI, blinded to the results of histopathology. The regions of interest were placed within the center of each test phantom to avoid edge artefacts and in the right liver avoiding large vessels and focal liver lesions. The absence of a liver lesion or large vessel in the region of interest was confirmed by visually comparing the region of interest positioned on the diffusion-weighted, T1- and T2-weighted images. The mean area for the regions of interest was $4.3 \pm 0.5 \mathrm{~cm}^{2}$ in the lipid emulsion-based phantoms and $18 \pm 6.8 \mathrm{~cm}^{2}$ in patients.

The apparent diffusion coefficient was measured using a monoexponential model [19] with the following equation:

$\mathrm{S}_{\mathrm{b}} / \mathrm{S}_{0}=\exp ^{(-\mathrm{bADC})}$

where $\operatorname{ADC}\left(10^{-3} \mathrm{~mm}^{2} / \mathrm{s}\right)$ is the apparent diffusion coefficient, $\mathrm{S}_{\mathrm{b}}$ the signal intensity for each $b$ value and $S_{0}$ the signal intensity at $b_{0}$.

The other diffusion coefficients were measured using a bi-exponential fit [19] with the following equation:

$\mathrm{S}_{\mathrm{b}} / \mathrm{S}_{0}=(1-\mathrm{f})^{\exp (-\mathrm{bD})}+\operatorname{fexp}^{\left(-\mathrm{b}\left(\mathrm{D}+\mathrm{D}^{*}\right)\right)}$

where, $f(\%)$ is the fraction of diffusion linked to microcirculation, $\mathrm{D}\left(10^{-3} \mathrm{~mm}^{2} / \mathrm{s}\right)$ the true diffusion coefficient and $\mathrm{D}^{*}\left(10^{-3} \mathrm{~mm}^{2} / \mathrm{s}\right)$ the perfusion-related diffusion coefficient.

The algorithms were implemented with purpose-built software running under the ROOT environment (ROOT 5.22, CERN, Geneva, Switzerland). The reproducibility of the diffusion parameter measurements has been reported previously [20].

\section{Statistical analysis}

The results are reported as mean \pm standard deviation. The correlation between the fat fraction and the diffusion coefficients for the lipid emulsion-based phantoms was calculated with Spearman correlation coefficients (r). The diffusion parameters of the patients with and without isolated liver steatosis were compared with the MannWhitney $U$ test after Bonferroni correction. $P \leq 0.01$ was considered to indicate a statistically significant difference. Statistical analysis was performed with the MedCalc software (MedCalc, Mariakerke, Belgium).

\section{Results}

The results of the diffusion parameter measurements in the phantoms are given in Table 1. The apparent and pure diffusion coefficients decreased from 2 and $2.1 \times 10^{-3} \mathrm{~mm}^{2} /$ $\mathrm{s}$ for a fat fraction of $0 \%$ to 1.42 and $1.49 \times 10^{-3} \mathrm{~mm}^{2} / \mathrm{s}$ for a fat fraction of $18 \%$ with fat suppression and from 2.04 and $2.1 \times 10^{-3} \mathrm{~mm}^{2} / \mathrm{s}$ for a fat fraction of $0 \%$, to 0.89 and $0.88 \times$ $10^{-3} \mathrm{~mm}^{2} / \mathrm{s}$ for a fat fraction of $18 \%$ without fat suppression, respectively. A strong inverse correlation was found between the fat fraction and apparent and pure diffusion coefficients either with fat suppression $(r=-0.98, P<0.0001$; $r=-0.97, P<0.0001$, respectively) or without fat suppression ( $r=-0.99, P<0.0001 ; r=-0.99, P<0.0001$, respectively). The measured perfusion fractions $(1.8 \pm 1 \%$ and $1 \pm 0.8 \%$ with and without fat suppression, respectively) and the perfusionrelated diffusion coefficients $\left(6.7 \pm 3.5 \times 10^{-3} \mathrm{~mm}^{2} / \mathrm{s}\right.$ and $3.6 \pm 2.4 \times 10^{-3} \mathrm{~mm}^{2} / \mathrm{s}$ ) were almost zero.

In patients with normal livers, the pure diffusion coefficient was significantly higher than in patients with isolated liver steatosis $\left(1.18 \pm 0.09 \times 10^{-3} \mathrm{~mm}^{2} / \mathrm{s}\right.$ versus $0.96 \pm 0.16 \times$ $10^{-3} \mathrm{~mm}^{2} / \mathrm{s}, P=0.005$ ) (Figs. 1, 2 and 3). The apparent diffusion coefficient, perfusion-related diffusion coefficient and perfusion fraction did not differ significantly between patients without and with isolated liver steatosis, but there was a small decreasing trend of the apparent diffusion coefficient in patients with liver steatosis (apparent diffusion coefficient: $1.41 \pm 0.14 \times 10^{-3} \mathrm{~mm}^{2} / \mathrm{s}$ versus $1.26 \pm$ $0.25 \times 10^{-3} \mathrm{~mm}^{2} / \mathrm{s}, \quad P=0.298$; perfusion-related diffusion coefficient: $99.9 \pm 2.18 \times 10^{-3} \mathrm{~mm}^{2} / \mathrm{s}$ versus $99.6 \pm 3.79 \times$ $10^{-3} \mathrm{~mm}^{2} / \mathrm{s}, P=0.754$; perfusion fraction: $21 \pm 2 \%$ versus $23 \pm 4 \%, P=0.431$, respectively).

\section{Discussion}

In this study performed in lipid emulsion-based phantoms and patients with and without isolated liver steatosis, we observed that the presence of fat droplets decreases the apparent and pure diffusion coefficients. In tissues containing both water and fat, it has been reported that slow diffusion may be explained by a restriction of water diffusion due to abundant lipids and by diffusion of mobile lipids, a slow phenomenon occurring at a rate that is 100 times slower than water diffusion [21, 22]. The respective influence of restricted water diffusion and slow lipid diffusion on the decrease of the diffusion parameters in steatosis is unknown. This influence will depend on the use of fat suppression. Without fat suppression, both restricted water diffusion and slow lipid diffusion may be observed, whereas with perfect fat suppression, only restricted water diffusion should be observed. Our findings in phantoms agree with these concepts, as the diffusion parameter measurements 
Table 1 Apparent $\left(\mathrm{ADC} \times 10^{-3} \mathrm{~mm}^{2} / \mathrm{s}\right)$ and pure $\left(\mathrm{D} \times 10^{-3} \mathrm{~mm}^{2} / \mathrm{s}\right)$ diffusion coefficients measured without and with fat suppression in lipid emulsion-based phantoms according to the fat fraction

\begin{tabular}{|c|c|c|c|c|c|c|c|c|c|c|c|}
\hline \multirow[t]{2}{*}{ Fat fraction $(\%)$} & \multirow[t]{2}{*}{0} & \multirow[t]{2}{*}{3} & \multirow[t]{2}{*}{5} & \multirow[t]{2}{*}{7} & \multirow[t]{2}{*}{9} & \multirow[t]{2}{*}{12} & \multirow[t]{2}{*}{14} & \multirow[t]{2}{*}{16} & \multicolumn{3}{|l|}{18} \\
\hline & & & & & & & & & & $r$ & $P$ \\
\hline ADC without fat suppression & 2.04 & 1.81 & 1.69 & 1.57 & 1.39 & 1.15 & 1.10 & 1.09 & 0.89 & -0.99 & $<0.0001$ \\
\hline ADC with fat suppression & 2.00 & 1.94 & 1.81 & 1.74 & 1.69 & 1.68 & 1.61 & 1.54 & 1.42 & -0.98 & $<0.0001$ \\
\hline D without fat suppression & 2.1 & 1.84 & 1.74 & 1.60 & 1.42 & 1.17 & 1.11 & 1.09 & 0.88 & -0.99 & $<0.0001$ \\
\hline D with fat suppression & 2.1 & 2.02 & 1.90 & 1.82 & 1.76 & 1.75 & 1.68 & 1.62 & 1.49 & -0.97 & $<0.0001$ \\
\hline
\end{tabular}

were higher with than without fat suppression. In vivo, perfect lipid signal suppression cannot be obtained because of multiple technical and biological reasons, including the fact that two of the fat spectrum peaks (olefinic acid at $5.3 \mathrm{ppm}$ and glycerol at $4.2 \mathrm{ppm}$ ) have frequencies very close to that of the water peak (at $4.7 \mathrm{ppm}$ ) [23]. These lipid peaks, representing $8-10 \%$ of the total fat spectrum, cannot be suppressed at clinical field strengths without also suppressing the water peak. Residual fat signal on fat suppressed diffusion-weighted images may decrease the measured diffusion parameters in two ways. First, slow lipid diffusion may be observed in these fat areas. Second, residual fat signal on high $b$-value diffusion-weighted images may artefactually decrease the measured diffusion parameters $[24,25]$. Regardless of the cause, our results show that isolated liver steatosis decreases the diffusion parameter measurements.

We observed similar results in liver steatosis and in phantoms closely mimicking this clinical condition. In contrast to the lipid phantom models previously described in the literature containing unmixed volumes of water and mineral oil in a container, imaged with an oblique imaging plane through the boundaries of both chemical environments to obtain different concentrations of water and fat [26, 27], our phantoms consisted of lipid emulsions, with lipid droplets

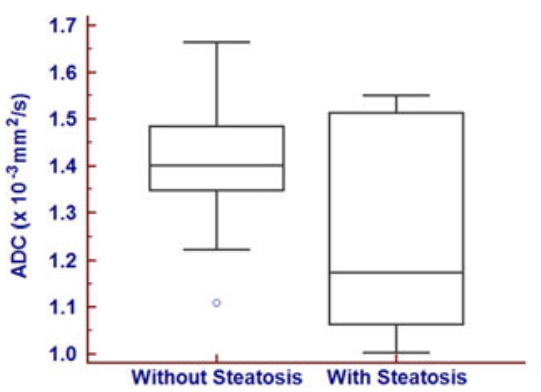

Fig. 1 Box and whisker plots showing the apparent (a) and pure (b) diffusion coefficients $\left(\times 10^{-3} \mathrm{~mm}^{2} / \mathrm{s}\right)$ according to the presence or absence of liver steatosis. Central box represents the values from the first to third quartiles and the middle line represents the median. The vertical line extends from the minimum to the maximum value within similar in size and concentration to the lipid inclusions in hepatocytes. Indeed, as mentioned in Materials and methods, the size of the lipid droplets in our phantoms $(0.6 \mu \mathrm{m})$ was close to that of intracellular fat droplets in liver steatosis (about $1 \mu \mathrm{m}$ ). Moreover, the fat percentages in the phantoms $(0-18 \%)$ covered a wide range of steatosis severity. It has previously been shown that the percentage of fat-containing hepatocytes is about 2.75 higher than the percentage of fat on a volume basis [28]. This means that a fat percentage of $18 \%$ in the phantoms relates to almost $50 \%$ fat-containing hepatocytes, i.e. moderate to severe steatosis according to the Brunt classification [18].

With respect to the clinical study and to the best of our knowledge, this is the first biopsy-proven study that reports the definite influence of isolated liver steatosis with no other pathological confounding factors on the diffusion parameters. Until now, limited and conflicting results have been published regarding the influence of steatosis on the diffusion parameters. In most studies, no significant influence was observed $[5,12,13]$. Recently, an inverse correlation between the apparent diffusion coefficient and hepatic fat fraction was reported in ex-vivo rat livers by Anderson et al. [14] and in patients by Poyraz et al. [15]. However, these two studies are limited by the absence of information about the specific influence of steatosis, inflammation and fibrosis

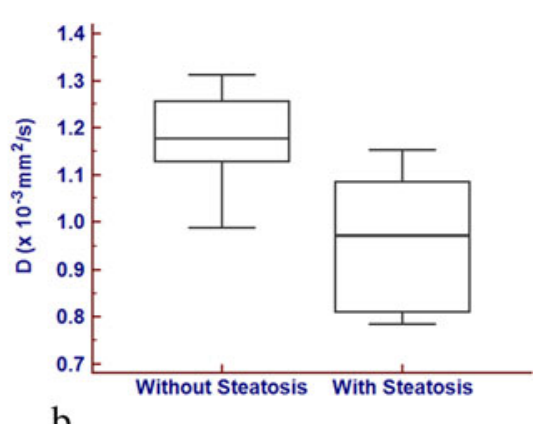

1.5 times the interquartile range. Outliers are represented as individual points. No significant difference in the apparent diffusion coefficient is observed between the two groups (a). The pure diffusion coefficient (b) is significantly lower in the steatotic group $(P=0.005)$ 


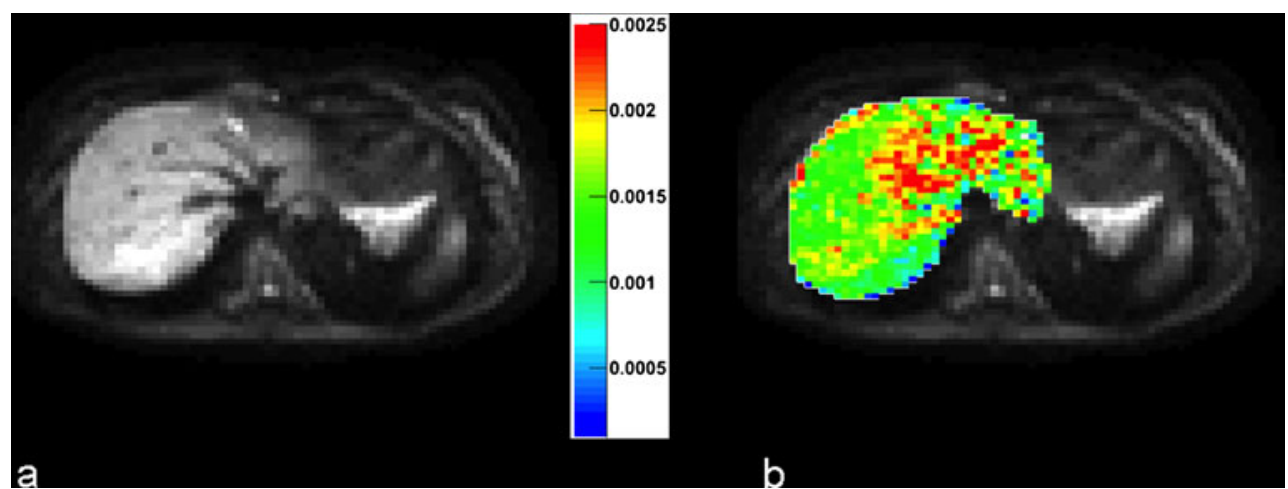

Fig. 2 Diffusion-weighted image at $b=10 \mathrm{~s} / \mathrm{mm}^{2}$ (a) and the same image with a superimposed parametric color map of the pure diffusion coefficient (b), with a scale in $\mathrm{mm}^{2} / \mathrm{s}$, of a 68 -year old woman without liver steatosis. The measured pure diffusion coefficient was $1.3 \mathrm{~mm}^{2} / \mathrm{s}$.

on the apparent diffusion coefficient measurements. Namely, in the retrospective clinical study by Poyraz et al. [15], liver biopsy was not obtained. In the ex-vivo study by Anderson et al. [14], histopathology was available, but a multivariate analysis of the influence of steatosis, inflammation and fibrosis on the diffusion measurements was not performed.

Moreover, except for the study of Lee et al. [13], only the apparent diffusion coefficient was measured in these previous works. The apparent diffusion coefficient, a compound parameter that includes influences from pure molecular diffusion and perfusion-related diffusion, may be less sensitive than the individual diffusion parameters to changes induced by steatosis or fibrosis, as shown in our study and that of Luciani et al. [9]. In nonalcoholic fatty liver disease, liver perfusion is decreased because of reduced sinusoidal volume [29]. However, we did not observe a decrease of the perfusion-related diffusion parameters in patients with liver steatosis. Several factors may explain this apparent discrepancy, such as the small number of patients with isolated liver steatosis in our study and the known difficulty of obtaining reliable results for the perfusion-related diffusion parameters [30]. Moreover, decreased perfusion has mainly been observed in steatohepatitis rather than in isolated steatosis,
The region of interest was placed in the right lobe, away from regions with apparent increases of diffusion. This apparent increase of diffusion in the left lobe may be explained by the flow in the large hepatic veins and transmitted cardiac motion

because sinusoidal compression and distortion in steatohepatitis are caused not only by fat deposits within hepatocytes, but also by hepatocyte hydropic ballooning, fibrosis of the space of Disse and leukocyte adhesion to the sinusoidal endothelium [29, 31].

Diffusion-weighted magnetic resonance imaging is not used to quantify liver fat, since other methods, such as proton spectroscopy and multi-echo gradient-echo imaging, are accurate for that purpose $[2,32]$. However, our results show that liver steatosis may decrease the measured diffusion parameters in chronic liver diseases. In addition to liver inflammation and decreased liver perfusion, which have previously been shown to decrease the diffusion parameters $[5,6,8$, 33], liver steatosis is hence a confounding factor when trying to stage liver fibrosis at diffusion-weighted MRI. Concomitant liver steatosis and fibrosis are often observed in chronic liver diseases, not only in nonalcoholic and alcoholic steatohepatitis but also in chronic viral hepatitis [34].

In our study, the decrease of diffusion parameters in patients with liver steatosis concerned the true diffusion coefficient, but not the apparent diffusion coefficient, calculated with a monoexponential approach using $11 \mathrm{~b}$ values ranging from 0 to $500 \mathrm{~s} / \mathrm{mm}^{2}$. It has been recently recommended to calculate the apparent diffusion coefficient with a
Fig. 3 Diffusion-weighted image at $b=10 \mathrm{~s} / \mathrm{mm}^{2}$ (a) and the same image with a superposed parametric color map of the pure diffusion coefficient (b), with a scale in $\mathrm{mm}^{2} / \mathrm{s}$, in a 52 -year old woman with liver steatosis. The measured pure diffusion coefficient $\left(0.82 \mathrm{~mm}^{2} / \mathrm{s}\right)$ was lower than that observed in a patient with normal liver

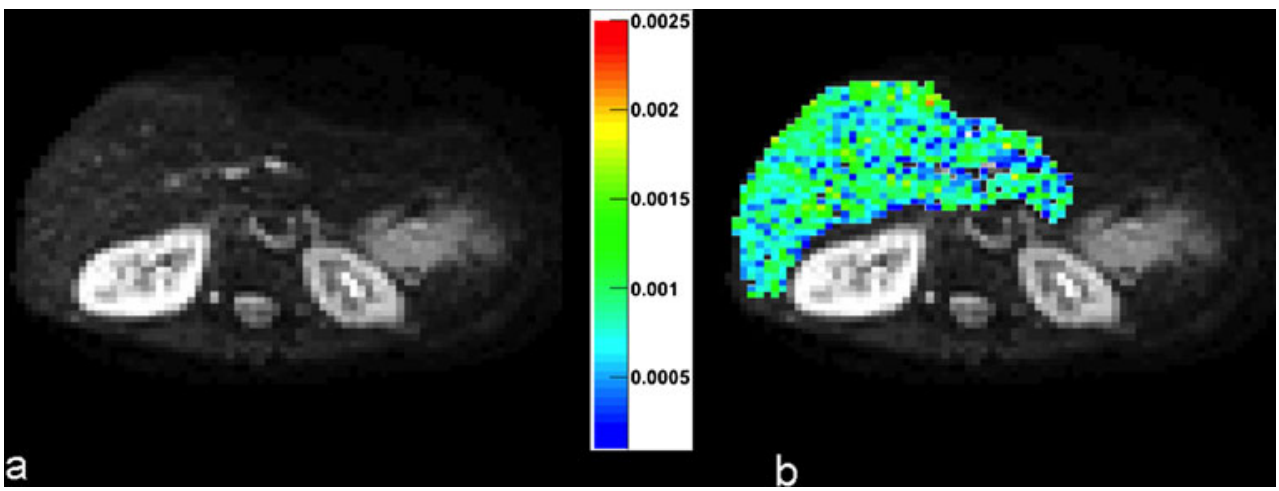


monoexponential approach using at least $2 \mathrm{~b}$ values $>100 \mathrm{~s} /$ $\mathrm{mm}^{2}$ to avoid the perfusion effect on the measured value [35]. In this case, the apparent diffusion coefficient equals the true diffusion coefficient and liver steatosis also decreases the apparent diffusion coefficient calculated with this method.

Our study has limitations. First, the number of included patients was small, because we excluded all patients with combined fat infiltration, iron overload, fibrosis and/or inflammation. The small cohort of patients with isolated liver steatosis might explain why we have not found a significant difference in the apparent diffusion coefficient between the two subgroups of patients since this composite parameter is less sensitive than the pure diffusion coefficient. Because of the small number of patients, we did not correlate the diffusion parameters with liver fat fractions. However, our phantom study suggests that an increasing fat fraction correlates with a decreasing diffusion. A larger prospective study in patients with isolated liver steatosis is needed to confirm these findings.

Secondly, we acquired free-breathing echo-planar diffusion-weighted imaging and physiological motion is always a concern when studying the microscopic displacement of protons. However, good reproducibility of the diffusion parameters was reported with free breathing or navigator-echo triggered sequences $[35,36]$.

In conclusion, our results show that the presence of fat droplets decreases the diffusion parameters and suggest that steatosis may have confounding effects when measuring the diffusion parameters at MRI.

Acknowledgements Helena S Leitão received a grant (SFRH/BD/ 33893/2009) from the Foundation for Science and Technology (FCT, Portugal).

\section{References}

1. Roulot D, Costes JL, Buyck JF et al (2011) Transient elastography as a screening tool for liver fibrosis and cirrhosis in a communitybased population aged over 45 years. Gut 60:977-984

2. Szczepaniak LS, Nurenberg P, Leonard D et al (2005) Magnetic resonance spectroscopy to measure hepatic triglyceride content: prevalence of hepatic steatosis in the general population. Am J Physiol Endocrinol Metab 288:E462-E468

3. Stefan N, Kantartzis K, Haring HU (2008) Causes and metabolic consequences of fatty liver. Endocr Rev 29:939-960

4. Wang Y, Ganger DR, Levitsky J, Sternick LA, McCarthy RJ, Chen ZE et al (2011) Assessment of chronic hepatitis and fibrosis: comparison of magnetic resonance elastography (MRE) and diffusion-weighted imaging (DWI). Am J Roentgenol 196:553561

5. Bonekamp S, Torbenson MS, Kamel IR (2011) Diffusionweighted magnetic resonance imaging for the staging of liver fibrosis. J Clin Gastroenterol 45:885-892

6. Taouli B, Chouli M, Martin AJ, Qayyum A, Coakley FV, Vilgrain V (2008) Chronic hepatitis: role of diffusion-weighted imaging and diffusion tensor imaging for the diagnosis of liver fibrosis and inflammation. J Magn Reson Imaging 28:89-95

7. Lewin M, Poujol-Robert A, Boelle P-Y et al (2007) Diffusionweighted magnetic resonance imaging for the assessment of fibrosis in chronic hepatitis C. Hepatology 46:658-665

8. Annet L, Peeters F, Abarca-Quinones J, Leclercq I, Moulin P, Van Beers BE (2007) Assessment of diffusion-weighted MR imaging in liver fibrosis. J Magn Reson Imaging 25:122-128

9. Luciani A, Vignaud A, Cavet M et al (2008) Liver cirrhosis: intravoxel incoherent motion MR imaging-pilot study. Radiology 249:891-899

10. Pais R, Pascale A, Fedchuck L, Charlotte F, Poynard T, Ratziu V (2011) Progression from isolated steatosis to steatohepatitis and fibrosis in nonalcoholic fatty liver disease. Clin Res Hepatol Gastroenterol 35:23-28

11. Sirlin CB (2009) Non invasive imaging biomarkers for steatosis assessment. Liver Transpl 15:1389-1391

12. d'Assignies G, Ruel M, Khiat A et al (2009) Noninvasive quantitation of human liver steatosis using magnetic resonance and bioassay methods. Eur Radiol 19:2033-2040

13. Lee JT, Liau J, Murphy P, Schroeder ME, Sirlin CB, Bydder M (2012) Cross-sectional investigation of correlation between hepatic steatosis and IVIM perfusion on MR imaging. Magn Reson Imaging 30:572-578

14. Anderson SW, Soto JA, Milch HN et al (2011) Effect of diffusion progression on liver apparent diffusion coefficient values in a murine model of NASH at 11.7 Tesla MRI. J Magn Reson Imaging 33:882-888

15. Poyraz AK, Onur MR, Kocakoç E, Ogur E (2012) Diffusionweighted MRI of fatty liver. J Magn Reson Imaging 35:1108-1111

16. Gimsberg HN, Goldberg IJ (2001) Disorders of the lipoprotein metabolism. In: Braunvald E, Fauci AS, Kasper DL, Hauser SL, Longo DL, Jameson JL (eds) Disorders of the intermediary metabolism. (Harrison's principals of internal medicine), 15th edn. Mc Graw-Hill, USA, p 2246

17. Crawford JM (2007) Basic mechanisms in hepatopathology. In: Burt AD, Portmann BC, Ferrell LD (eds) MacSween's pathology of the liver, 5th edn. Churchill Livingstone Elsevier, Philadelphia, Philadelphia, $\mathrm{p} 85$

18. Brunt EM, Tiniakos DG (2010) Histopathology of nonalcoholic fatty liver disease. World J Gastroenterol 16:5286-5296

19. Le Bihan D, Breton E, Lallemand D, Aubin M-L, Vignaud J, Laval-Jeantet M (1988) Separation of diffusion and perfusion in intravoxel incoherent motion MR imaging. Radiology 168:497-505

20. Kim SY, Lee SS, Byun JH et al (2010) Malignant hepatic tumors: short-term reproducibility of apparent diffusion coefficients with breath-hold and respiratory-triggered diffusion-weighted MR imaging. Radiology 255:815-823

21. Ababneh ZQ, Beloeil H, Berde CB, Ababneh AM, Maier SE, Mulkern RV (2009) In vivo lipid diffusion coefficient measurements in rat bone marrow. Magn Reson Imaging 27:859-864

22. Qiao Y, Ronen I, Viereck J, Ruberg FL, Hamilton JA (2007) Identification of atherosclerotic lipid deposits by diffusionweighted imaging. Arterioscler Thromb Vasc Biol 27:1440-1446

23. Reeder SB, Cruite I, Hamilton G, Sirlin CB (2011) Quantitative assessment of liver fat with magnetic resonance imaging and spectroscopy. J Magn Reson Imaging 34:729-749

24. Wenkel E, Geppert C, Schulz-Wendtland R et al (2007) Diffusion weighted imaging in breast MRI: comparison of two different pulse sequences. Acad Radiol 14:1077-1083

25. Baron P, Dorrius MD, Kappert P, Oudkerk M, Sijens PE (2010) Diffusion-weighted imaging of normal fibroglandular breast tissue: influence of microperfusion and fat suppression technique on the apparent diffusion coefficient. NMR Biomed 23:399-405

26. O'Reagan DP, Callaghan MF, Wylezinska-Arridge M, Fitzpatrick J, Naoumova RP, Hajnal JV et al (2008) Liver fat content and T2*: 
simultaneous measurements by using breath-hold multiecho MR imaging at $3.0 \mathrm{~T}$-feasibility. Radiology 247:550-557

27. Hussain HK, Chenevert TL, Londy FJ, Gulani V, Swanson SD, McKenna BJ et al (2005) Hepatic fat fraction: MR imaging for quantitative measurement and display — early experience. Radiology 237:1048-1055

28. Marsman H, Matsushita T, Dierkhising R, Kremers W, Rosen C, Burgart L (2004) Assessment of donor liver steatosis: pathologist or automated software. Hum Pathol 35:430-435

29. Farrell GC, Teoh NC, McCuskey RS (2008) Hepatic microcirculation in fatty liver disease. Anat Rec 291:684-692

30. King MD, van Bruggen N, Busza AL, Houseman J, Williams SR, Gadian DG (1992) Perfusion and diffusion MR imaging. Magn Reson Med 24:288-301

31. McCuskey RS, Ito Y, Robertson GR, McCuskey MK, Perry M, Farrell GC (2004) Hepatic microvascular dysfunction during evolution of dietary steatohepatitis in mice. Hepatology 40:386393
32. Yokoo T, Bydder M, Hamilton G et al (2009) Nonalcoholic fatty liver disease: diagnostic and fat-grading accuracy of low-flip-angle multiecho gradient-recalled-echo MR imaging at $1.5 \mathrm{~T}$. Radiology 251:67-76

33. Annet L, Materne R, Danse E, Jamart J, Horsmans Y, Van Beers BE (2003) Hepatic flow parameters measured with MR imaging and Doppler US: correlations with degree of cirrhosis and portal hypertension. Radiology 229:409-414

34. Paradis V, Bedossa P (2008) Definition and natural history of metabolic steatosis: histology and cellular aspects. Diabetes Metab 34:638-642

35. Padhani AR, Liu G, Mu-Koh D et al (2009) Diffusion-weighted magnetic resonance imaging as a cancer biomarker: consensus and recommendations. Neoplasia 11:102-125

36. Patel J, Sigmund EE, Rusinek H, Oei M, Babb JS, Taouli B (2010) Diagnosis of cirrhosis with intravoxel incoherent motion diffusion MRI and dynamic contrast-enhanced MRI alone and in combination: preliminary experience. J Magn Reson Imaging 31:589-600 